Kébili (Tunisie méridionale), le 10 novembre 1906. Je possède en outre un autre mâle et une femelle entièrement semblables à l'exemplaire décrit. D'après MM. P. Mabille et G. Hampson, cette espèce nouvelle appartient certainement, par sa nervulation et son faciès, au genre Polia.

\title{
Descriptions d'un genre nouveau et d'une espèce nouvelle de Myriapodes de France
}

\author{
par H.-W. BröLemann.
}

Macrosternodesmus, nov. gen. - Hanches des pattes copulatrices trapues, larges et montées sur des poches trachéennes médiocrement développées, comme le seraient celles des Leptodesmiens. Patte copulatrice divisée en deux rameaux. Rameau séminal se détachant de la patte à l'extrémité distale du tibia.

Corps composé de 19 segments. Formule des pores : 5, 7, 9, 10, 12, 13, 10 et suivants. Lames ventrales des somites plus longues que de coutume, déterminant un plus grand écartement des pattes ambulatoires. Dernier article des pattes ambulatoires plus long que tous les autres. Très petites formes.

Macrosternodesmus palicola, nov. sp. - Long. : environ 3,כ0 4 mill.; larg. : environ 0,30-0,40 mill. - Corps entièrement décoloré, très ténu, un peu déprimé, composé de 19 segments, avec 28 (

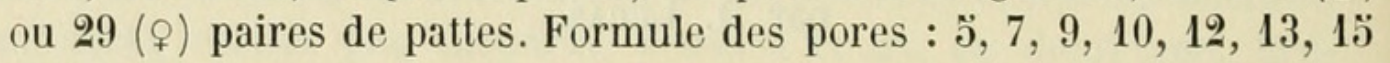
et suivants.

Tète largement dépassée latéralement par le tronc des mandibules. Lèvre supérieure faiblement incisée, l'échancrure comblée par les dentelures usuelles. Surface densément pileuse, notamment en avant. Antennes de longueur moyenne, fortement claviformes; 亏ّe article très court, conique, portant au bord antérieur quelques bâtonnets olfactifs; $6^{e}$ article très large, plus long et plus densément vêtu de poils que les précédents, avec un fort faisceau de nombreux et longs bâtonnets olfactifs; $7^{\mathrm{e}}$ article en tronc de cône, bien dégagé; 4 bàtonnets subaigus à l'extrémité.

Tous les téguments présentent une structure réticulée due, semblet-il, à de minuscules verrues plates, régulièrement distribuées sur toute la surface; sur les prozonites, ces verrues sont orientées longitudinalement, de façon à former des rangées transversales. Sur aucun 
écusson on ne reconnaît de mamelons caractérisés; la surface des écussons est sịmplement inégale, avec, de distance en distance, des granulations portant des poils très courts. Ces granulations forment trois rangées de huit granulations chacune; celles de la rangèe postérieure font légèrement saillie sur le bord de l'écusson. Les deux granulations médianes de la rangée antérieure sont placées en avant de l'alignement des autres. Les rangées sont séparées l'une de l'autre par une dépression transversale.

Premier écusson en demi-cercle, beaucoup plus étroit que la tête. Écussons du tronc à carènes peu développées, à angle antérieur arrondi, à angle postérieur droit, nullement prolongé, tronqué sur les écussons porteurs de foramina; bord latéral des carènes interrompu par trois ou quatre fines dentelures piligères.

Avant-dernier somite dégagé, aussi long que le précédent. Somite préanal long, en forme de grand crochet rapidement aminci et courbé vers le sol; sa pointe tronquée dépasse le niveau du bord des valves anales, qui sont cependant très proéminentes.

Lames ventrales longues et larges, divisées transversalement en deux moitiés subégales par un sillon profond; inermes. L'écartement des pattes est plus grand que la longueur des tibias correspondants (sur les somites du milieu du corps); en outre les deux hanches du même còté de chaque somite sont écartées l'une de l'autre, au lieu d'être contiguës, comme c'est généralement le cas. - Suture pleuro-ventrale indistincte.

Pattes de dimensions normales; le dernier article est le plus long de tous.

Pattes copulatrices d'un type particulier. Hanches médiocrement allongées, assez larges, pourvues d'un grand crochet et montées sur une poche trachéenne bien conformée. Fémoroïde très court et élargi, portant, sur sa face externe, trois grandes soies mêlées aux soies usuelles. Tibia très modelé, comprimé latéralement, donnant naissance, à son extrémité distale, à deux rameaux. Le rameau séminal, graduellement aminci et deux fois sinué, porte sur son arête externe une lamelle translucide, subrectangulaire, dont l'angle inférieur est prolongé en triangle aigu (fig. 1); il se termine par une tigelle droite, finement fourchue à son extrémité. Le rameau secondaire est robuste, droit ou à peine un peu cintré dans les trois premiers quarts de sa longueur; il est coudé avant l'extrémité et se

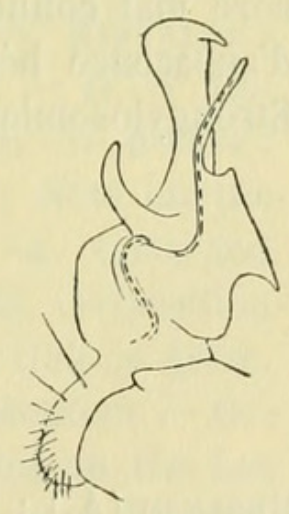

Fig. 1. - Profil externe de la patte copulatrice. 


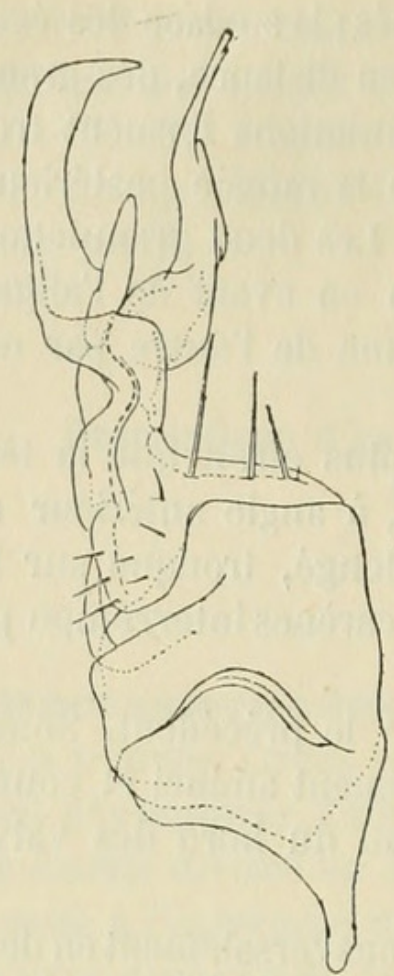

Fig. 2. - Face antérosupérieure de la même, plus grossie.

termine en pointe rapidement amincie (fig. 2); ce rameau porte, à sa base externe, un long et robuste crochet, à pointe émoussée. La rainure séminale suit un parcours à silhouette tortueuse dans le tibia, puis, passant dans l'aisselle des deux rameaux, va aboutir dans la tigelle du rameau séminal.

Cette espèce est adulte en automne. C'est en novembre et décembre (1907) qu'ont été trouvés des mâles adultes et des jeunes des deux sexes, dans un jardin de Pau, dans la terre d'une plate-bande longeant un mur couvert de Lierre mort. Le 22 février suivant, quelques rares femelles ont pu être recueillies, mais les mâles semblaient avoir disparu.

Cette espèce n'accuse pas d'affinités bien définies; la forme de ses hanches nous amènerait à la classer dans les Leptodesmiens (mihi), si elle ne s'en écartait par les proportions des articles des pattes ambulatoires (caractère de peu de valeur, il est vrai) et aussi (ce qui est pluś important) par la forme de ses pattes copulatrices. On sait que, chez les Leptodesmiens, le rameau séminal est une émanation du fémur; ici, au contraire, c'est de l'extrémité du tibia qu'il se détache, comme c'est le cas chez les Strongylosomiens. C'est sans doute dans la microfaune circumméditerrannéenne, qui est encore mal connue, qu'il faut chercher les analogies de cette forme d'apparence hétéroclite, que nous classerons provisoirement avec les Strongylosomiens.

\section{Bulletin bibliographique.}

Berlese (A.) : Gli Insetti, loro organizzazione, Sviluppo, abitudini e rapporti coll' uomo, I, 3, 1908, pp. 713-800; Milan.*

Chevreux (Ed.) : Description de deux nouvelles espèces d'Amphipodes des parages de Monaco; (Bull. Instr. océan.) 1908, 8 p., fig.* 


\section{$2 \mathrm{BHL}$ Biodiversity Heritage Library}

1908. "Description d'un genre nouveau et d'une espece nouvelle de Myriapodes de France." Bulletin de la Société entomologique de France 1908, 94-96. https://doi.org/10.5962/bhl.part.5606.

View This Item Online: https://www.biodiversitylibrary.org/item/37020

DOI: https://doi.org/10.5962/bhl.part.5606

Permalink: https://www.biodiversitylibrary.org/partpdf/5606

\section{Holding Institution}

Smithsonian Libraries

\section{Sponsored by}

Smithsonian

\section{Copyright \& Reuse}

Copyright Status: NOT_IN_COPYRIGHT

This document was created from content at the Biodiversity Heritage Library, the world's largest open access digital library for biodiversity literature and archives. Visit BHL at https://www.biodiversitylibrary.org. 